\title{
An X-shooter survey of star forming regions: Low-mass stars and sub-stellar objects *
}

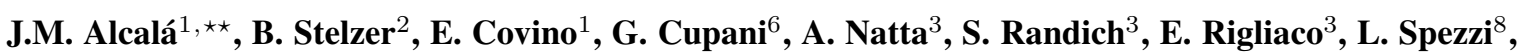 \\ L. Testi ${ }^{3}$, F. Bacciotti ${ }^{3}$, R. Bonito ${ }^{10,2}$, S. Covino ${ }^{7}$, E. Flaccomio ${ }^{2}$, A. Frasca ${ }^{4}$, D. Gandolfi ${ }^{8}$, F. Leone ${ }^{11,4}$, \\ G. Micela ${ }^{2}$, B. Nisini ${ }^{5}$, and E. Whelan ${ }^{9}$ \\ 1 INAF - Osservatorio Astronomico di Capodimonte, Via Moiariello 16, I-80131 Napoli, Italy \\ 2 INAF - Osservatorio Astronomico di Palermo, Piazza del Parlamento 1, 90134 Palermo, Italy \\ 3 INAF - Osservatorio Astrofisico di Arcetri, Largo E.Fermi 5, I-50125 Firenze, Italy \\ ${ }^{4}$ INAF - Osservatorio Astrofisico di Catania, via S. Sofia, 78, 95123 Catania, Italy \\ 5 INAF - Osservatorio Astronomico di Roma, Via di Frascati 33, 00040, Monteporzio Catone, Italy \\ ${ }^{6}$ INAF - Osservatorio Astronomico di Trieste, via Tiepolo 11, 34143 Trieste, Italy \\ 7 INAF - Osservatorio Astronomico di Brera, via Emilio Bianchi 46, 23807 Merate (LC), Italy \\ 8 Research and Scientific Support Department, ESA-ESTEC, PO Box 299, 2200 AG Noordwijk, The Netherlands \\ ${ }^{9}$ Laboratoire dAstrophysique de Grenoble, UMR 5571, BP 53, 38041 Grenoble Cedex 09, France \\ 10 Dip. di Scienze Fis. ed Astron., Sez. Astronomia, Univ. di Palermo, .zza del Parlamento 1, 90134 Palermo, Italy \\ 11 Dip. di Fisica e Astronomia Sezione Astrofisica, Univ. di Catania, Via S. Sofia 78, I95123 Catania, Italy
}

Received 2011 Feb 2, accepted 2011 Feb 7

Published online 2011 Mar 17

Key words accretion, accretion disks - ISM: jets and outflows - stars: formation - stars: low-mass, brown dwarfs stars: pre-main sequence $-\mathrm{T}$ Tauri stars

We present preliminary results of our X-shooter survey in star forming regions. In this contribution we focus on subsamples of young stellar and sub-stellar objects (YSOs) in the Lupus star forming region and in the TW Hya association. We show that the $\mathrm{X}$-shooter spectra are suitable for conducting several parallel studies such as YSO + disk fundamental parameters, accretion and outflow activity in the very low-mass (VLM) and sub-stellar regimes, as well as magnetic activity in young VLM YSOs, and Li abundance determinations. The capabilities of X-shooter in terms of wide spectral coverage, resolution and limiting magnitudes, allow us to assess simultaneously the accretion/outflow, magnetic activity, and disk diagnostics, from the UV and optical to the near-IR, avoiding ambiguities due to possible YSO variability.

(C) 2011 WILEY-VCH Verlag GmbH \& Co. KGaA, Weinheim

\section{Introduction}

The data from new surveys in the mid-IR show that the paradigm of star formation via core-collapse and the formation of an accretion disk that survives for large part of the Pre-Main Sequence (PMS) phase very likely applies to all low-mass objects, down to planetary-masses (Natta et al. 2006; Allers et al. 2006; Alcalá et al. 2008). These recent studies have shown that basically all low-mass objects are surrounded by disks during their early PMS evolution and show signs of mass accretion from the disk. However, disk properties (i.e. disk geometry, gas chemistry, dust composition and grain size, mass-accretion and mass-loss rates, etc.) show huge and unexplained differences both among stars of similar mass and over the mass spectrum.

A reliable determination of the disk properties requires an assessment of the complete spectral energy distribution (SED), from optical to $\mathrm{mm}$ wavelengths, and an accurate knowledge of the physical parameters of the central object.

\footnotetext{
$\star$ Based on observations collected at the European Southern Observatory, Chile, under Programmes 084.C-0269 and 085.C-0238.

$\star \star$ Corresponding author: alcala@oacn.inaf.it
}

Since the stellar and sub-stellar physical parameters are often not known with sufficient accuracy, the characteristics of the disks cannot be derived in a reliable way. This hampers our ability to address the issue of planet formation, which depends on disk properties, such as the mass accretion rate $\left(\dot{M}_{\mathrm{acc}}\right)$, the ejection of jets and winds, and the growth and settling of dust. In particular, $\dot{M}_{\text {acc }}$ is one of the key parameters during the PMS evolution. The total mass accumulated during the proto-stellar phase depends on this parameter, as well as on the timescale of accretion which for low-mass YSOs has been estimated to be of the order of $0.55 \mathrm{Myr}$ (Evans et al. 2009).

Various methods have been used in the literature to measure mass accretion rates (e.g. Gullbring et al. 1998; Muzerolle et al. 2001; Herczeg et al. 2008). In particular, it has been shown that the mass accretion rate, $\dot{M}_{\text {acc }}$, derived from veiling and UV excess correlates with several emission lines, e.g. $\mathrm{H} \alpha$ emission $10 \%$ width, near-IR hydrogen line luminosity, line flux of the Ca II infrared triplet (e.g. Muzerolle et al. 1998), underlining the importance of these spectral features as accretion diagnostics. These correlations, applied to large samples of low-mass YSOs, were shown to 
hold down to the brown dwarf (BD) regime (e.g. Natta et al. 2004; Mohanty et al. 2005; Herczeg et al. 2008), providing a relationship between $\dot{M}_{\text {acc }}$ and mass of the central object (Muzerolle et al. 2005; Natta et al. 2006) although with a large scatter (of about 2 dex) for a given mass. The main caveat of the calibrations used to determine $\dot{M}_{\text {acc }}$ is the nonsimultaneity of photometry and spectral line measurements, complicated by the fact that emission lines can trace both strong winds and mass accretion. Moreover, at low accretion rates, the lines used as accretion diagnostics may be significantly contaminated by the chromospheric activity of the central object. Therefore, the derivation of mass-accretion and mass-loss rates and, in general, the reliability of magnetospheric accretion models tested in T Tauri stars are uncertain when applied to the very-low mass (VLM) regime.

In order to address the issues above in a systematic way, a spectroscopic survey on homogeneous samples of young stellar objects (YSOs) is crucial. The X-shooter capabilities offer a unique opportunity to obtain a complete picture of YSOs, allowing us to assess simultaneously the diagnostics for accretion and outflows, disks, magnetic activity, and to define the stellar photospheric flux from the UV and optical to the near IR. To this aim, we started an X-shooter survey in galactic star forming regions (SFRs), as a part of the Italian $\mathrm{X}$-shooter GTO. Our programme is conceived in such a way as to address a number of relevant issues in low-mass star and BD formation by studying properly selected samples of YSOs with disks in different formation environments; henceforth, our definition of a 'YSO' includes both young low-mass stars and BDs. In this contribution we present preliminary results for sub-samples of YSOs in the Lupus SFR and the TW Hya association, while a detailed study of a young BD in the $\sigma$ Ori cluster is reported in Rigliaco et al. (2011a,b) and Rigliaco et al. (2011c).

\section{Sample, observations and data reduction}

The targets have been selected according to the following criteria: i) regions of low extinction; ii) regions that are nearby $(\mathrm{d}<500 \mathrm{pc})$ with many VLM YSOs $\left(\mathrm{M}<0.2 \mathrm{M}_{\odot}\right)$, but covering a mass spectrum as ample as possible, including several more massive (up to $2 \mathrm{M}_{\odot}$ ) objects; iii) YSOs well surveyed in as many photometric bands as possible: mainly Spitzer (IRAC and MIPS) surveys and complementary UV, optical and millimeter data available. Using these criteria, targets mainly in the $\sigma$ Ori, Chamaeleon, Lupus and TW Hya regions have been selected. The stellar and substellar populations of these regions are found in different evolutionary stages and environments, and are thus ideal to study the evolution of the above properties and their dependence on the environment.

Our programme foresees to investigate some hundred YSOs in the aforementioned regions. Table 1 summarizes the statistics of targets observed so far in each region, and in parenthesis the number of objects that we expect to observe
Table 1 Statistics of observations as of January 2011.

\begin{tabular}{lccl}
\hline Region & $\begin{array}{c}\text { Age }^{*} \\
(\mathrm{Myr})\end{array}$ & No. of YSOs & Date \\
\hline$\sigma$ Ori & $1.5-3.5$ & $\begin{array}{c}10 \\
(12)\end{array}$ & $\begin{array}{l}\text { Dec 2009 } \\
\text { (Jan 2011) }\end{array}$ \\
Lupus & $5-10$ & 11 & Apr 2010 \\
TW Hya & $10-20$ & $\begin{array}{l}4 / 9 \\
(5)\end{array}$ & $\begin{array}{l}\text { Mar 2010/Apr 2010 } \\
\text { (Jan 2011) }\end{array}$ \\
\hline
\end{tabular}

* The range of age for each region was taken from Reipurth (2008).

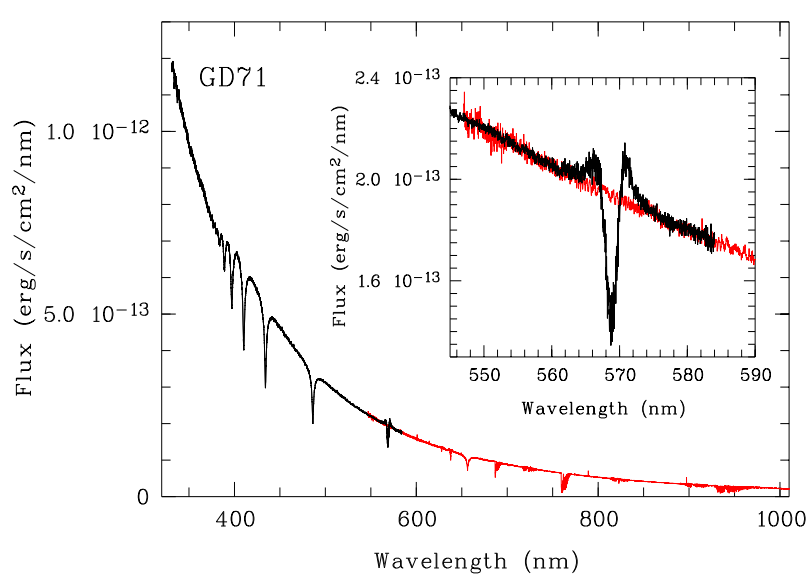

Fig. 1 (online colour at: www.an-journal.org) Flux-calibrated spectrum of the flux standard GD71 observed in the December 2009 run. The inset shows the detail of the good match in flux between the UVB (black) and VIS (red) arms. The absorption-like feature at $\sim 569 \mathrm{~nm}$ in the UVB arm is a well known instrumental artifact, possibly due to the dichroic.

in the forthcoming run of January 2011. Thus we expect to have about half of our sample observed by early 2011 .

Several among the $\sim 100$ objects were selected in each region as photospheric templates based on their spectral types and the lack of strong accretion signatures. The gravity of such templates will be similar to that of the accreting YSOs of the corresponding region, providing a good match of the YSOs photospheric spectrum. In addition, two YSOs with outflows, observed in the April 2010 run, will be discussed in Sect. 5. The adopted observational strategy is such that the highest resolution allowed by X-shooter (using a $\sim 0.5^{\prime \prime}$ slit) has been exploited to observe the bright objects, most of which in the TW Hya association, whereas most of the targets in the other regions were observed with the $1^{\prime \prime}$ slit width. Nodding mode was used for most of the observations. More details on the observational strategy of the survey will be given in our forthcoming papers.

The data reduction was performed independently for each spectrograph arm using the $\mathrm{X}$-shooter pipeline version 1.1.0. Special care was put in the reduction of visual binaries and targets with outflows for which 'stare-mode' without background subtraction was used. The background subtraction for these objects was performed separately with IRAF. 


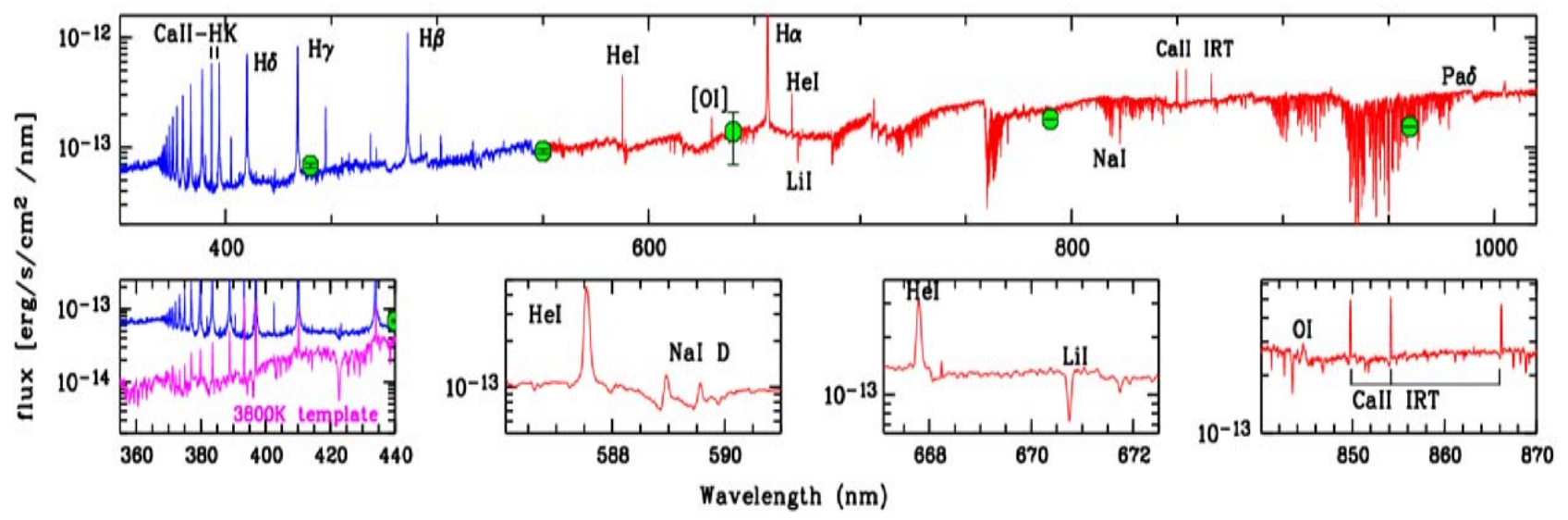

Fig. 2 (online colour at: www.an-journal.org) Example of the UVB (blue) and VIS (red) spectrum of the YSO Sz130 in the Lupus IV cloud, with some details of spectral regions (lower panels). The big green circles represent the flux as derived from de-reddened $B V R I Z$ photometry ( $\left.A_{V}=0.6 \mathrm{mag}\right)$ available in Merín et al. (2008).

The 'flexcomp' command was used in order to correct for flexures of the instrument. This is crucial for the measurements of radial velocity (see Sect. 3.1).

Flux calibration for the UVB and VIS arms has been achieved using context LONG within MIDAS. For this purpose, a response function was derived by interpolating the counts/standard-flux ratio of the flux standards, observed the same night as the objects (under photometric sky conditions), with a third-order spline function, after airmass correction. The response function was then applied to the 1D spectra. An example of the flux calibrated UVB + VIS spectrum of the standard star GD71, observed during the December 2009 run, is shown in Fig. 1. Noteworthy is the good flux match between the two arms. A flux calibration of the IR arm has not been performed yet.

As an example for our targets, the flux-calibrated UVB and VIS spectrum of the YSO Sz 130 in Lupus is shown in Fig. 2. Many accretion diagnostics such as the Balmer lines (up to $\mathrm{H} 24$ ), and the $\mathrm{He}$ I and $\mathrm{Ca}$ II (H, K, and IR triplet) are clearly seen in emission, and likewise the Balmer Jump. In addition, the $\operatorname{Li}$ I $\lambda 670.78 \mathrm{~nm}$ absorption line is very well detected and resolved from nearby $\mathrm{Fe}$ I lines. Note also that the $\mathrm{Na}$ I D lines $\lambda \lambda 589.0,589.6 \mathrm{~nm}$ are very well resolved and in emission.

There is a very good agreement between the flux of the spectrum and the one derived from $B V R I Z$ photometric measurements available in the literature. We have verified this for many of our targets. Therefore, we can rely on the flux calibration of the X-shooter spectra to measure the flux of the many diagnostics, as will be discussed in Sect. 4.

\section{Physical parameters of YSOs}

In a first attempt to characterize the accretion of the YSO sample observed so far, we adopt some of the basic physical parameters of the YSOs from the literature. For the time being, we adopt spectral type, effective temperature

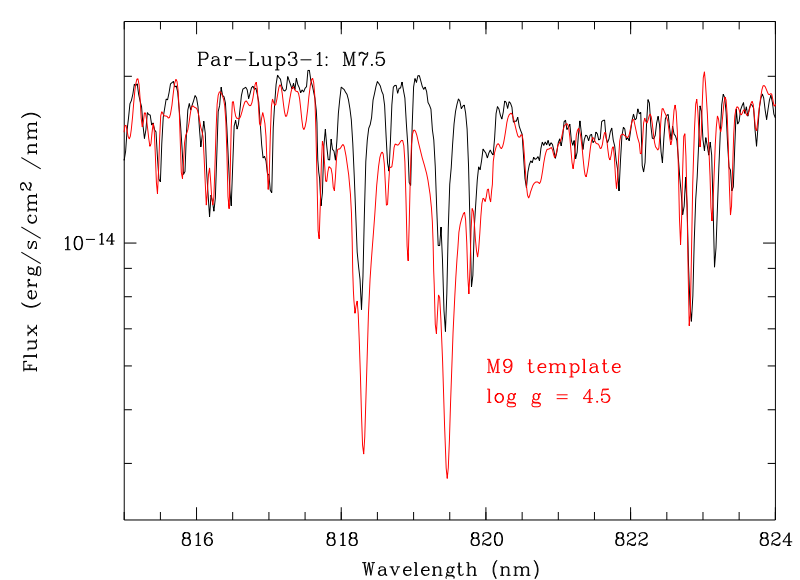

Fig. 3 (online colour at: www.an-journal.org) Detail of the Xshooter spectrum of the YSO Par-Lup-3-1 (spectral type M7.5) displaying the Na I doublet at $\lambda \lambda 818.3,819.5 \mathrm{~nm}$. Overplotted is the spectrum of a M9V template (J104814.2-395606) with $\log g=4.5$. The narrower Na I line wings of Par-Lup-3-1 with respect to those of the template, indicate a lower surface gravity, $\log g \sim 3.5$.

( $\left.T_{\text {eff }}\right)$ and optical extinction $\left(A_{V}\right)$ values from Merín et al. (2008) and Mentuch et al. (2008) for the YSOs in Lupus and TW Hya, respectively. Previous studies have shown that the analysis of different spectral ranges may yield different spectral types (e.g. Alcalá et al. 2006; Allers et al. 2006; Testi 2009; Comeron et al. 2010). Thus, the use of the entire $\mathrm{X}$-shooter spectrum, from the UV to near-IR, will be crucial for the lowest mass objects, for which spectral type is still poorly explored. Therefore, in the near future, we intend to re-determine spectral types, $T_{\text {eff }}$ and $A_{V}$ homogeneously by a bi-parametric $\chi^{2}$ minimization of the flux-calibrated 1D $\mathrm{X}$-shooter spectra to a grid of spectral type templates with similar spectral resolution (e.g. Frasca et al. 2003; Alcalá et al. (2006). The $\log g$ values can be derived with a similar approach as the $\chi^{2}$ minimization, using gravity-sensitive 


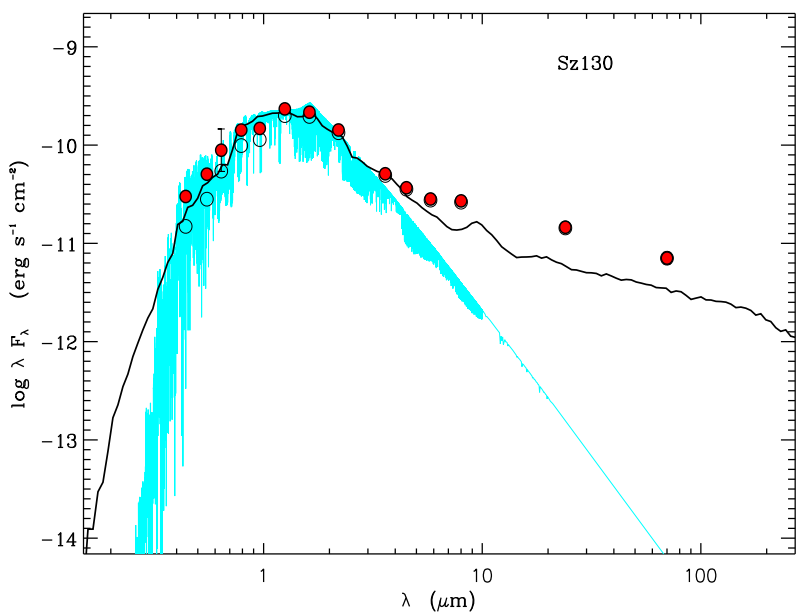

Fig. 4 (online colour at: www.an-journal.org) Observed (open circles) and de-reddened (red dots) SED of the YSO Sz130. We used the photometry reported in Merín et al. (2008) to derive the SED. The model spectrum of the same $T_{\text {eff }}$ and $\log g$ as $S z 130$, scaled by the object's distance $(150 \mathrm{pc})$ and radius $\left(1.12 \mathrm{R}_{\odot}\right)$, is overplotted in blue. The best accretion-disk model + star SED fit is represented by the black continuous line.

photospheric lines. For instance, Fig. 3 shows that the $\mathrm{Na}$ I $\lambda \lambda 818.3,819.5 \mathrm{~nm}$ lines of the YSO Par-Lup-3-1 are shallower with respect to those of an object with similar spectral type, but higher gravity. Although the line core can be filled in by chromospheric or accretion-driven emission, the line wings, strongly sensitive to gravity, are clearly narrower in the YSO spectrum. A caveat of the $\chi^{2}$ minimization procedure is the lack of templates with a good coverage both in $T_{\text {eff }}$ and $\log g$, in a wide spectral range. This calls for the necessity of X-shooter templates with a good grid resolution, both in $T_{\text {eff }}$ and $\log g$.

The luminosity is computed as follows: first, a model spectrum of the same $T_{\text {eff }}$ as the YSO is scaled by the YSO distance and then fitted to the de-reddened optical SED. The reddening is independently derived by comparing observed colors with the intrinsic ones corresponding to the same $T_{\text {eff }}$ as the object. The luminosity is then derived by integration of the model spectrum. Figure 4 shows the example of the de-reddened SED of Sz130. Mass and age are then derived by comparison with theoretical PMS evolutionary tracks on the HR diagram (e.g. Spezzi et al. 2008). Disk parameters can be derived by SED fitting to the de-reddened SED, using accretion disk models (e.g. Robitaille 2006). The mass accretion rate resulting from the disk SED fit is $\dot{M}_{\text {acc }}=2.2 \times 10^{-9} \mathrm{M}_{\odot} \mathrm{yr}^{-1}$, in good agreement with the one derived from accretion diagnostics (see Sect. 4), which is $\dot{M}_{\text {acc }}=1.6 \times 10^{-9} \mathrm{M}_{\odot} \mathrm{yr}^{-1}$. The disk inner radius resulting from the SED fit is 5.3 times the stellar radius, i.e. consistent with the average value for most YSO accretors (see Sect. 4).

Although the YSOs radius is normally calculated from Stefan-Boltzmann's law, we have explored the possibility

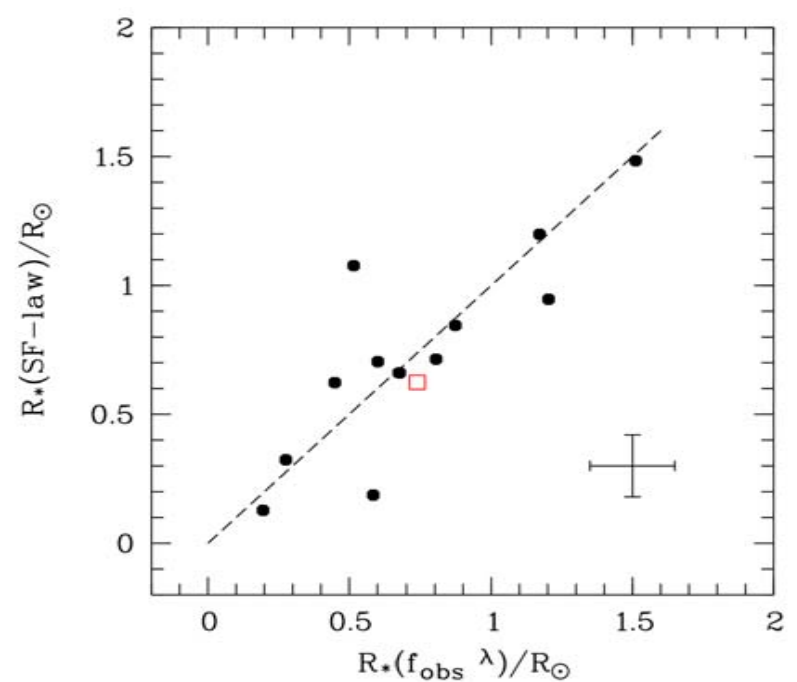

Fig. 5 (online colour at: www.an-journal.org) The radii derived from Stefan-Boltzmann's law versus those derived using Eq. (1) for the Lupus YSO sample. The open square represents the young brown-dwarf $\sigma$ Ori 500 (Rigliaco et al. 2011a,b). The average error bars are overplotted.

to derive this parameter from the flux-calibrated $\mathrm{X}$-shooter spectra, $f_{\text {obs }}^{\lambda}$, using the relationship

$R_{\star}\left(f_{\mathrm{obs}}^{\lambda}\right)=d \times\left\langle\frac{f_{\mathrm{obs}}^{\lambda}}{F_{\star}^{\lambda}\left(T_{\mathrm{eff}}, \log g\right)}\right\rangle^{1 / 2}$,

where $d$ is the object's distance and $F_{\star}^{\lambda}\left(T_{\text {eff }}, \log g\right)$ is the photospheric continuum flux at the star's surface. The latter can be obtained from model atmospheres (e.g. Dusty AMES models by Chabrier et al. 2000 and Allard et al. 2001) for the appropriate object's $T_{\text {eff }}$ and $\log g$ values. The average indicated in Eq. (1) can be done in principle throughout the entire spectral range, but in order to minimize effects due to telluric lines, as well as emission lines of the observed spectrum, we select a $50 \mathrm{~nm}$ spectral range centered at about $820 \mathrm{~nm}$. The good agreement between the radius calculated in this way with the one derived from Stefan-Boltzmann's law (see Fig. 5) confirms the reliability of our flux calibration, as well as of the assumption of $\log g=3.5$ for the Lupus YSOs and $\sigma$ Ori 500. The assumed distances for the latter are $140 \mathrm{pc}$ and $360 \mathrm{pc}$, respectively.

\subsection{Radial and rotational velocities}

$\mathrm{X}$-shooter allows the determination of relatively accurate radial velocity (RV) and projected rotational velocity $(v \sin i)$ by means of cross-correlation techniques, as well as by fitting synthetic models. Using the spectra obtained at the highest resolution we have estimated that instrument flexures may introduce systematic shifts of the order of $0.2 \AA$ in the wavelength calibrated spectra, which means $\sim 8 \mathrm{~km} \mathrm{~s}^{-1}$ at $\sim 700 \mathrm{~nm}$. Therefore, correction for flexures are critical in order to measure RV with $\mathrm{X}$-shooter. Telluric lines may also be considered as a useful check of wavelength calibration. Finally, an example of the determination of $v \sin i$ of 


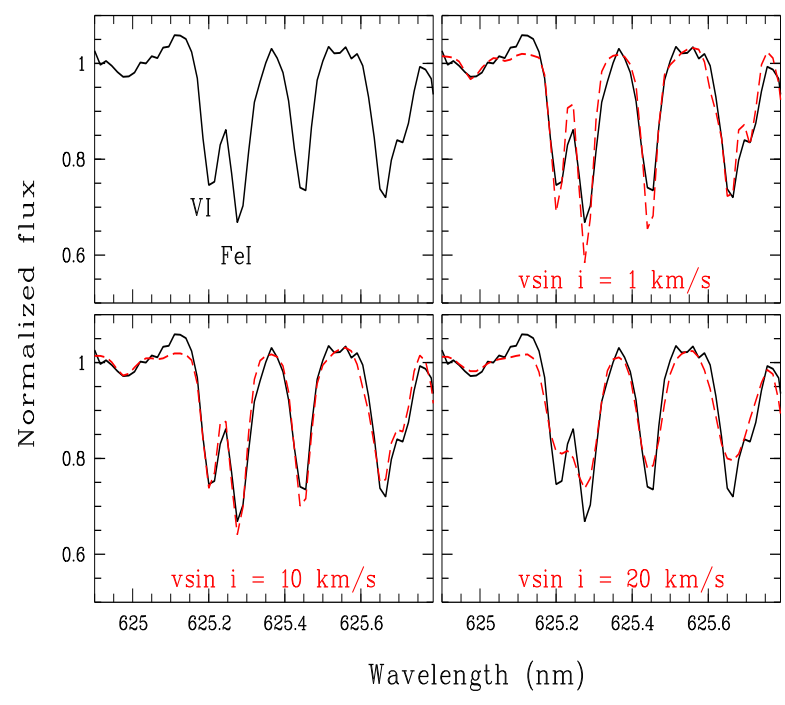

Fig. 6 (online colour at: www.an-journal.org) Fit of a synthetic model to the X-shooter spectrum of CD-36 7429A showing the V I and $\mathrm{Fe} \mathrm{I}$ lines. In three of the four panels a synthetic spectrum with $T_{\text {eff }}=4250 \mathrm{~K}$ and $\log g=3.5$ convolved with a rotation profile at three $v \sin i$ values is overlaid (dashed lines). The best fit gives a $v \sin i \approx 10 \pm 4 \mathrm{~km} \mathrm{~s}^{-1}$, which can be compared with the value of $7.5 \pm 3 \mathrm{~km} \mathrm{~s}^{-1}$ derived by Torres et al. (2006) from a FEROS spectrum.

CD 36-7429A, by fitting a synthetic spectrum to the V I and Fe I lines, is shown in Fig. 6. From our analysis, we find that the precisions on $\mathrm{RV}$ and $v \sin i$, that can be achieved with $\mathrm{X}$-shooter when used at its maximum resolution, are $\sim 1 \mathrm{~km} \mathrm{~s}^{-1}$ and $\sim 5 \mathrm{~km} \mathrm{~s}^{-1}$, respectively. Therefore, we conclude that $\mathrm{X}$-shooter allows to derive kinematical properties with sufficient accuracy for membership studies.

\section{Mass accretion rate}

In the magnetospheric accretion model the mass accretion rate, $\dot{M}_{\text {acc }}$, is related to the accretion luminosity, $L_{\text {acc }}$, by

$\dot{M}_{\mathrm{acc}}=\left(1-\frac{R_{\star}}{R_{\mathrm{in}}}\right)^{-1} \frac{L_{\mathrm{acc}} R_{\star}}{G M_{\star}} \approx 1.25 \frac{L_{\mathrm{acc}} R_{\star}}{G M_{\star}}$,

where $R_{\star}$ and $M_{\star}$ are the YSO radius and mass, respectively, and $R_{\text {in }}$ is the YSO inner-disk radius (Hartmann 1998). The latter corresponds to the distance at which the disk is truncated - due to the stellar magnetosphere - and from which the disk gas is accreted, channelled by the magnetic field lines. In previous works it has been assumed that $R_{\text {in }}$ is $\sim 5 R_{\star}$. Indeed from results of YSO disk SED modelling in previous works (Alcalá et al. 2008) we find that for most accretors $R_{\mathrm{in}} / R_{\star} \sim 5$, which justifies the approximation in the second step of Eq. (2).

The accretion luminosity $L_{\mathrm{acc}}$ is directly linked to the excess UV emission and veiling. As such, it can be derived from modelling of the Balmer Jump using the observed UVB spectra, following the same approach described by Valenti et al. (1993) and Herczeg \& Hillenbrand (2008).
The latter authors provide linear relationships between $L_{\text {acc }}$ (estimated from Balmer Jump modelling) and the luminosity, $L_{\text {line }}$, of many accretion diagnostics such as the Balmer lines, and the $\mathrm{He} \mathrm{I}$ and $\mathrm{Ca} I \mathrm{II}(\mathrm{H}, \mathrm{K}$, and IR triplet) emission lines. Thus, the accretion luminosity can be computed from a relation of the type $\log L_{\mathrm{acc}}=a \log L_{\text {line }}+b$, where the coefficient $a$ and term $b$ can be adopted from Table 16 by Herczeg \& Hillenbrand (2008). Such linear relationships can be used to provide a first and quick estimate of $L_{\mathrm{acc}}$, and hence of $\dot{M}_{\text {acc }}$ from Eq. (2). ${ }^{1}$

The line luminosity is given by $L_{\text {line }}=4 \pi d^{2} F_{\text {line }}$, where $d$ is the distance and $F_{\text {line }}$ is the extinction-corrected absolute flux of the line, calculated by simple integration of the flux calibrated spectrum. We use several different lines to determine $\dot{M}_{\text {acc }}$ using the empirical relations with $L_{\text {acc }}$ as described above. As expected, the $L_{\text {acc }}$ and $\dot{M}_{\text {acc }}$ values derived from different diagnostics are in fairly good agreement (see Fig. 7). Thus, the simultaneous observation of various accretion diagnostics for a given YSO in a single spectrum allows us to compute an average $\dot{M}_{\text {acc }}$, minimizing the scatter due to possible variability.

The mass accretion rate can also be estimated from the relationships by Natta et al. (2004), Mohanty et al. (2005), and Muzerolle et al. (1998), using as diagnostics the width of the $\mathrm{H} \alpha$ line at $10 \%$ of the line peak, the flux of the Ca II $\lambda$ 866.2nm line at the star's surface and the $\mathrm{Pa} \beta$ and $\mathrm{Br} \gamma$ recombination lines in the near-IR, respectively. However, in order to draw conclusions on the resulting $\dot{M}_{\text {acc }}$ values using different methods and diagnostics, a statistically significant sample of YSOs should be analysed. Therefore, comparisons of the results from different methods are deferred to a future paper, once our $\mathrm{X}$-shooter survey will be completed.

Although the sample of YSOs investigated so far in our survey is still too limited for drawing statistically significant statements, we can compare their position in the $\dot{M}_{\text {acc }}$ vs. $M_{\star}$ diagram with that of other YSOs (see Fig. 8). While the Lupus YSOs follow a trend similar to those in Taurus and $\sigma$ Ori, the majority of the TW Hya YSOs do not. The latter is consistent with the TW Hya YSOs being more evolved, and their emission lines being mainly of chromospheric origin. In this sense, the derived $\dot{M}_{\text {acc }}$ values for most of these objects must be considered as upper limits, because the emission lines in their spectrum trace mainly chromospheric activity. While the line fluxes of the TW Hya objects are rather high (see Fig. 7), the line luminosities follow the same correlations as for the Lupus accretors. This would mean that the chromospheric luminosity of the TW Hya objects is distributed among the different emission lines in the same way as the accretion luminosity of the Lupus accretors, which is interesting. Most importantly, and despite the low number statistics, our $\mathrm{X}$-shooter survey already provides several

1 Rigorously speaking, one should model the Balmer Jump using the X-shooter UVB spectra to derive $L_{\text {acc }}$, as done for instance for the young BD $\sigma$ Ori-500 (Rigliaco et al. 2011a,b). However, this goes beyond the scope of the present paper, but will be done in the future for the complete YSO X-shooter sample. 

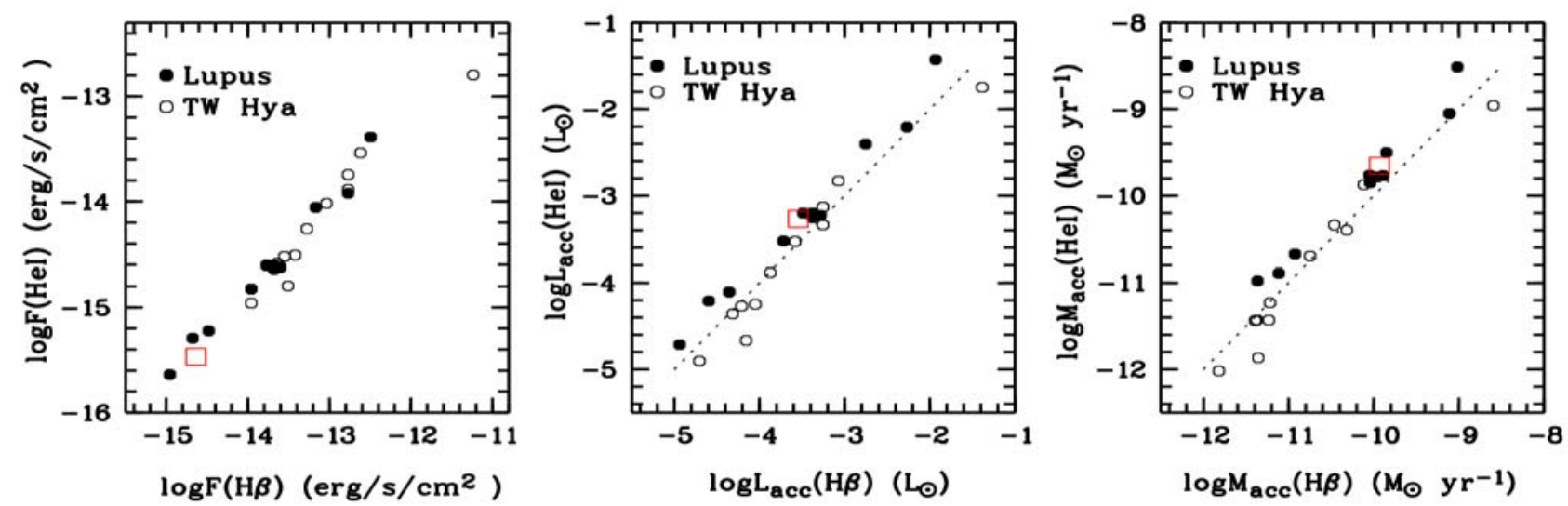

Fig. 7 (online colour at: www.an-journal.org) Results from the X-shooter run of April 2010 of targets in TW Hya (circles) and Lupus (dots). Examples of correlations between line fluxes, $L_{\mathrm{acc}}$, and $\dot{M}_{a c c}$ derived using the $\mathrm{He} \mathrm{I}(\lambda 5876 \AA)$ and $\mathrm{H} \beta$ lines as diagnostics. The square represents the young BD $\sigma$ Ori-500 (Rigliaco et al. 2011a,b).

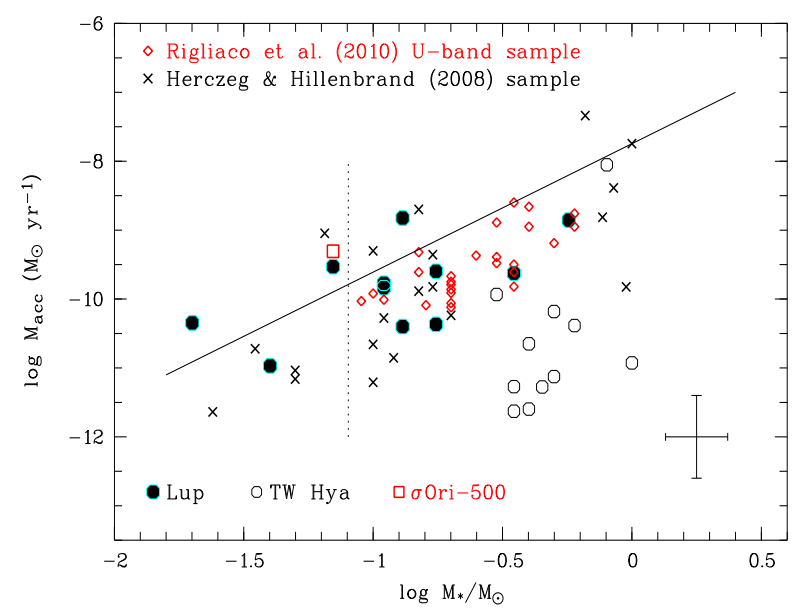

Fig. 8 (online colour at: www.an-journal.org) The $\dot{M}_{\text {acc }}$ vs. $M_{\star}$ scatter plot for the same objects as in Fig. 7, but using the average $\dot{M}_{\text {acc }}$, derived using the different diagnostics. The average errors on $\log \dot{M}_{\text {acc }}$ and $\log M_{\star}$ are indicated on the lower right. The continuous line represents the $\dot{M}_{\text {acc }} \propto M_{\star}^{+1.9}$ relationship derived by Herczeg \& Hillenbrand (2008), while the dotted line represents the sub-stellar boundary.

accreting BDs for which $\dot{M}_{\text {acc }}$ is derived. These objects represent key targets for our forthcoming studies of accretion in the VLM and sub-stellar regimes, and more of them are going to be observed in future $\mathrm{X}$-shooter runs.

\section{Outflows}

Accretion is also related to outflow activity at different mass regimes; winds and jets have been detected also in sub-stellar objects (e.g. Whelan et al. 2009; Rigliaco et al. 2011a,b). The study of such outflows enables to elucidate the relationship between mass ejection and accretion, and test the universality of the star formation mechanism as a function of (sub-) stellar mass. Our survey includes a few YSOs as a pilot project to exploit the performances of X-shooter to investigate outflows. Our analysis indicates that the spectrum of ESO $\mathrm{H} \alpha-574$ is very rich of emission lines tracing the jet in all the investigated wavelength range. The RV difference of the outflow components at each line is very low, suggesting an edge-on disk. The very wide range of the spectral mapping allows us to identify immediately high excitation lines in the outflow. the Position-velocity diagrams obtained for numerous lines indicates that the gas excitation is higher in the vicinity of the source (first 2-3") and decreases in the outer knots. For Par-Lup3-4 a bipolar jet is detected in several lines, the brightest being [O I] $\lambda \lambda 630,636.3 \mathrm{~nm},[\mathrm{~N} \mathrm{II}] \lambda \lambda 654.8,658.3 \mathrm{~nm}$, and [S II] $\lambda \lambda 671.6,673.0 \mathrm{~nm}$. The emission is definitely extended in [S II]. Also, in the case of Par-Lup3-4, a low RV difference is measured between the components of the jet, consistent with an edge-on disk. The latter result is in very good agreement with the findings from SED modelling of Par-Lup3-4 by Huelamo et al. (2010). The details and results of our investigation of these two outflows will be published elsewhere (Bacciotti et al. 2011).

\section{Concluding remarks}

Based on X-shooter spectroscopy, first estimates of mass accretion rate have been derived for several young low-mass stellar and sub-stellar objects in three regions representing different environments and evolutionary stages. The preliminary results on the physical parameters and mass accretion rates of the so far observed YSOs are very encouraging. The potential of $\mathrm{X}$-shooter resides indeed in the possibility to measure simultaneously many photospheric and accretion diagnostics, minimizing errors due to possible variability. In addition, the $\mathrm{X}$-shooter resolution allows to derive kinematical properties with sufficient accuracy for membership studies. The YSOs investigated so far in Lupus follow the same trend $\left(\dot{M}_{\text {acc }} \propto M_{\star}^{\gamma}\right.$, where $\left.\gamma \approx+2.0\right)$ in the $\dot{M}_{\text {acc }}$ vs. $M_{\star}$ diagram as previously found for other YSOs in Taurus and $\rho$ Oph. Such trend seems to extend to the sub-stellar regime, 
indicating that the accretion properties of sub-stellar objects are similar to those of low-mass YSOs. However, there are two important issues that still deserve further study. First, the distinction between the chromospheric and accretion origin of the different emission line diagnostics, at the lowest accretion rate levels; second, a fine tuning of YSO physical parameters, by using more accurate distance determinations. The latter issue is crucial when deriving $\dot{M}_{\text {acc }}$ and $M_{\star}$. In fact, in the study by Rigliaco et al. (2011a) a flattening of the $\dot{M}_{\text {acc }}$ vs. $M_{\star}$ relationship is found, with a value of $\gamma$ close to 1 , when extending the relationship to higher masses. An analogous effect seems to be seen when adopting an updated distance determination for the YSOs in the $\rho$ Oph cloud (Randich, private communication). The results of our pilot programme on YSO outflows, demonstrate that $\mathrm{X}$-shooter may provide new insights on the study of such objects. The wide spectral range and sensitivity of the instrument allow us to immediately determine the different excitation states of the gas along the outflows. Large $\mathrm{X}$-shooter surveys of SFRs in the near future will provide data sets representing benchmarks for studies of low-mass star and BD formation, as well as for the investigations of YSOs atmospheres, and evolutionary models of PMS stellar and sub-stellar evolution.

Acknowledgements. We would like to dedicate this contribution to R. Pallavicini. We thank the meeting organizers for their kind invitation to write the manuscript. We thank V. D'Odorico, P. Goldoni and A. Modigliani for their help with the X-shooter pipeline and F. Getman for the pipeline installation. We thank the ESO staff, in particular F. Patat for help in OB preparation and C. Martayan for support in the observations. We acknowledge an anonymous referee for helpful comments. Financial support from INAF is also acknowledged.

\section{References}

Alcalá, J.M., Spezzi, L., Frasca, A., et al.: 2006, A\&A 453, 1 Alcalá, J.M., Spezzi, L., Chapman, N., et al.: 2008, ApJ 676, 427 Allers, K.N., et al.: 2006, ApJ 644, 364

Bacciotti, F., et al.: 2011, in prep.

Comerón, F., Testi, L., Natta, A.: 2010, A\&A 522, 47

Evans, N.J., et al.: 2009, ApJ 181, 321

Frasca, A., et al.: 2003, A\&A 405, 149

Gullbring, E., et al.: 1998, ApJ 492, 323

Harmann, L.: 1998, Accretion Processes in Star Formation, Cambridge Univ. Press, Cambridge

Herczeg, G., Hillenbrand, L.: 2008, ApJ 681, 594

Huelamo, N., et al.: 2010, A\&A 523, 42

Mentuch, E., et al.: 2008, ApJ 689, 1127

Merín, B., Spezzi, L., Alcalá, J.M., et al.: 2008, ApJS 177, 551

Mohanty, S., Jayawardhana, R., Basri, G.: 2005, ApJ 626, 498

Muzerolle, J., Hartmann, L., Calvet, N.: 1998, AJ 116, 2965

Muzerolle, J., Calvet, N., Hartmann, L.: 2001, ApJ 550, 944

Muzerolle, J., et al.: 2005, ApJ 625, 906

Natta, A., Testi, L., Randich, S.: 2006, A\&A 452, 245

Natta, A., Testi, L., Muzerolle, J., et al.: 2004, A\&A 424, 603

Reipurth, B.: 2008, Handbook of Star Forming Regions, The Southern Sky ASP Monograph Publications

Rigliaco, E., et al.: 2011a, A\&A 525, 47

Rigliaco, E., et al.: 2011b, A\&A 526, 6

Rigliaco, E., Natta, A., Testi, L., Randich, S., Covino, E., Alcalá, J.M.: 2011c, AN 332, 249

Robitaille, T.P., et al.: 2006, ApJS 167, 256

Spezzi, L., et al.: 2008, ApJ 680, 1295

Testi, L.: 2009, A\&A 503, 639

Torres, C.A.O., et al.: 2006, A\&A 460, 695

Valenti, J.A., Basri, G., Jones, C.M.: 1993, AJ 106, 2024

Whelan, E., et al. : 2009, ApJ 706, 1054 